\title{
Una regla de predicción clínica ¿anticipa el diagnóstico de la faringitis estreptocóccica en niños de 2 a 15 años?
}

\author{
Lorena Karzulovic', Patricia García ${ }^{2}$, Aniela Wozniak², Luis Villarroeß', \\ Tamara Hirsch ${ }^{1}$, Ida Concha ${ }^{1}$, Silvia Catalán ${ }^{1}$ y Lorena Cifuentes ${ }^{1}$
}

\begin{abstract}
'Departamento de Pediatría, Escuela de Medicina, Pontificia Universidad Católica de Chile. Santiago, Chile. Departamento de Laboratorios Clínicos, Escuela de Medicina, Pontificia Universidad Católica de Chile. Santiago, Chile. ${ }^{3}$ Departamento de Salud Pública, Escuela de Medicina, Pontificia Universidad Católica de Chile. Santiago, Chile.
\end{abstract}

Conflicto de interés: Ninguno que declarar.

Fuente de Financiamiento Departamento de Pediatría de la

Pontificia Universidad Católica de Chile y el Departamento de Laboratorios Clínicos de la Pontificia Universidad Católica de Chile.

Recibido: 27 de diciembre de 2017 Aceptado: 27 de julio de 2018

Correspondencia a: Lorena Cifuentes Águila Lorena.cifuentes@uc.cl

\section{Does a clinical prediction rule anticipate the diagnosis for streptococcal pharyngitis in children aged 2 to 15 ?}

Background: The etiology of a streptococcal pharyngitis must be documented by laboratory techniques to avoid unnecessary antimicrobial treatment, but this strategy increases cost for the patient. Available scores applied in children or adults are imperfect. Aim: To develop a clinical prediction rule to aid the diagnostic process of streptococcal pharyngitis (SP) in children in a low-resource setting. Methods: Three hundred and eighteen patients aged 2 to 15 years who were evaluated for suspected SP at the Pediatric Emergency Department and the Pediatric Ambulatory Unit of Red Salud UC-Christus entered the study. A throat culture and a rapid antigen detection test for Streptococcus pyogenes were obtained from each patient. Data were analyzed for possible clinical predictors of SP with univariate and multiple regression analyses. Results: Seventy-three cases of SP were diagnosed $(23.9 \%)$. In the univariate analysis, fever was inversely associated with SP $(\mathrm{p}=0.002)$. Odynophagia, palatal petechiae, and season of the year (autumn and winter) were positively associated with $\operatorname{SP}(p=0.007, p<0.001$ and $p=0.03$ respectively). In multiple regression analysis the models did not have sufficient power to predict streptococcal etiology. Conclusion: Clinical predictors, even those systematically included in clinical prediction rules, did not show sufficient predictive power to safely include or exclude SP in this setting, and thus, it is necessary to improve access to confirmatory tests.

Keywords: Streptococcus pyogenes, pharyngitis, clinical prediction rule, children.

Palabras clave: Streptococcus pyogenes; faringitis; regla de predicción clínica; niños.

\section{Introducción}

a realización de un diagnóstico generalmente está asociada a un importante nivel de incertidumbre. Un diagnóstico preciso es esencial para un tratamiento apropiado y un pronóstico exacto. Las Reglas de Predicción Clínica (RPC) son herramientas de diagnóstico que incluyen las características clínicas ponderadas de la historia del paciente, examen físico y/o exámenes complementarios, mostrando las probabilidades de un diagnóstico o pronóstico específico ${ }^{1,2}$. Su objetivo es disminuir la incertidumbre y promover una toma de decisiones segura y eficiente mediante la selección más precisa de los pacientes que necesitan pruebas adicionales para hacer un diagnóstico.

Streptococcus grupo A o Streptococcus pyogenes es la causa bacteriana más común de faringitis aguda y representa aproximadamente 17 a $37 \%$ de los casos de faringitis aguda en niños ${ }^{3-6}$. La importancia de un diagnóstico y tratamiento precisos de la faringitis estreptocóccica (FE) es prevenir sus complicaciones supurativas, no supurativas y su transmisión a terceros ${ }^{7}$.

La faringitis viral y bacteriana comparten muchos sig- nos y síntomas, por lo que es muy difícil incluir o excluir un diagnóstico de $\mathrm{FE}$ basado únicamente en los hallazgos clínicos $^{8}$. Dado que el diagnóstico clínico no es confiable, la mayoría de las guías publicadas para el diagnóstico y manejo recomiendan la confirmación de laboratorio de la etiología estreptocóccica con pruebas rápidas de detección de antígeno (PRDA) y/o cultivo faríngeo, que se consideran las pruebas de referencia para el diagnóstico de $\mathrm{FE}^{9-11}$. Desafortunadamente, la confirmación del laboratorio agrega un costo adicional al proceso de diagnóstico de FE. Además, las pruebas indiscriminadas de todos los niños con dolor de garganta conducirían a un aumento de los costos de atención médica y molestias adicionales. Sin embargo, tratar a niños sin confirmación de laboratorio aumenta el uso innecesario de antimicrobianos.

En Chile, las PRDA y cultivo faríngeo no están fácilmente disponibles, particularmente en el sistema público de salud. Este hecho, además del temor a posibles complicaciones de la FE, han llevado a un uso excesivo de antimicrobianos. Se estima que, en nuestro medio, 12 a $50 \%$ de los pacientes con faringitis aguda reciben antibioterapia innecesariamente ${ }^{4-5}$.

Por lo tanto, es razonable especular sobre si los ha- 
llazgos clínicos organizados de una manera particular en una RPC, se pueden utilizar para una mejor asignación de recursos. Existen varios estudios publicados sobre RPC para FE, y muchos de ellos son en niños ${ }^{12-16}$. Sin embargo, sólo unos pocos han sido validados apropiadamente para ser usados con confianza en diferentes poblaciones y escenarios. Se sabe que esta falta de validación modifica la eficacia diagnóstica informada de las $\mathrm{RPC}^{17}$. Además, ningún estudio ha sido desarrollado o validado en una población latinoamericana o similar a la nuestra. Por lo tanto, buscamos identificar predictores clínicos de FE en nuestra población y validar una RPC para ayudar en el diagnóstico de FE.

\section{Material y Método}

\section{Pacientes y entorno}

Estudio transversal realizado en el Departamento de Emergencia Pediátricas y la Unidad Ambulatoria de la Pontificia Universidad Católica de Chile. El Departamento de Emergencia Pediátrica recibe aproximadamente 25.000 visitas cada año, y la Unidad Ambulatoria Pediátrica recibe 10.000 visitas anuales. El tamaño de la muestra se calculó utilizando la ecuación de Freeman ${ }^{18}$ y modificada por Peduzzi ${ }^{19}$ para modelos de regresión logística. Considerando que el modelo final podría tener un máximo de cinco niveles en el puntaje (es decir, $\mathrm{K}=5$ ítems), y asumiendo que alrededor de $\mathrm{P}=20 \%$ de los niños presentarán $\mathrm{FE}$, el cálculo del tamaño muestral fue $\mathrm{n}>=10 * \mathrm{~K} / \mathrm{P}=10 * 5 / 0,2=250$ individuos. Se incluyeron en el estudio los niños de 2 a 15 años con sospecha clínica de FE y que aceptaron participar. Se excluyeron los pacientes inmunodeprimidos y los que habían recibido antimicrobianos en los últimos 7 días. Se obtuvo el consentimiento informado de todos los participantes, que fue firmado por los adultos responsables del niño en el momento del reclutamiento, y el asentimiento informado en niños mayores de 7 años. El protocolo fue aprobado por el Comité de Ética en Investigación de la Facultad de Medicina de nuestra Universidad.

\section{Diseño del estudio}

El presente estudio representa la fase de derivación en el desarrollo de un protocolo para el diagnóstico de FE mediante reacción de polimerasa en cadena. En esta fase, se identifican los predictores del resultado de interés, en este caso, FE. Los factores con poder predictor se agrupan en la regla, que a su vez se usa como prueba de diagnóstico. Se adoptaron los siguientes resguardos metodológicos para reducir el posible sesgo y maximizar la validez interna del estudio de test diagnóstico actual ${ }^{20}$ :

- Un espectro adecuado de gravedad de la enfermedad: la muestra incluyó niños con faringitis, en los que existía incertidumbre diagnóstica entre la etiología viral y bacteriana, para asemejarse a la práctica clínica tanto como sea posible y asegurar la aplicabilidad adecuada del estudio. Por lo tanto, los criterios de inclusión y exclusión fueron seleccionados, con la intención de excluir pacientes con una evidente mayor probabilidad de FE (p. ej., erupción escarlatiniforme) para evitar una sobreestimación de la precisión de la prueba.

- Una comparación ciega entre la prueba y un estándar de oro independiente y aceptado. El cultivo faríngeo es el estándar de oro actual para el diagnóstico de la FE y es aceptado en todo el mundo. Las PRDA también es una técnica aceptada para hacer un diagnóstico de $\mathrm{FE}^{11}$. La interpretación de los cultivos faríngeos se realiza en forma rutinaria por el personal del laboratorio de microbiología; ocurre en un lugar diferente de los sitios de reclutamiento y sin acceso a las características clínicas del paciente. Los médicos no tuvieron conocimiento de los resultados en el momento de la evaluación clínica y del registro en el formulario de estudio.

- La PRDA no está incluida como parte de cultivo faríngeo; por lo tanto, la PRDA se considera una evaluación independiente del estándar de oro.

- La ausencia de sesgo de verificación: el cultivo faríngeo (estándar de oro) se realizó en todos los niños incluidos en el estudio, independientemente del resultado de la prueba clínica.

\section{Recolección de datos}

Para los pacientes incluidos, los médicos asistentes completaron un formulario especialmente diseñado para el estudio que incluía el nombre del paciente y el número de identificación nacional, así como una lista de características clínicas. Las características clínicas se enumeraron con recuadros "sí" o "no" para facilitar el proceso de registro, e incluyeron los siguientes ítems: fiebre (autoinformada y/o medida), odinofagia, mialgias, síntomas catarrales, tos, dolor abdominal, vómitos, contacto con un caso de FE, ganglios linfáticos submandibulares sensibles, exudado amigdalino, petequias palatinas, halitosis y rinolalia.

\section{Pruebas de laboratorio}

Después de la evaluación clínica, se obtuvieron dos muestras faríngeas de cada niño para realizar un cultivo faríngeo y PRDA para S. pyogenes. Dos hisopos se frotaron vigorosamente y en forma simultánea sobre la faringe posterior y las amígdalas. El primer hisopo se introdujo en medio de transporte Stuart (CulturetteTM, Beckton Dickinson Microbiology Systems, Cockeysville, MD) y se inoculó antes de una hora en agar sangre y placas de agar Polyvitex (bioMérieux, Marcy l'Etoile, Francia). Las placas se incubaron a $35^{\circ} \mathrm{C}$ en $6-8 \%$ de $\mathrm{CO}_{2}$ y se 
leyeron a las 24 y $48 \mathrm{~h}$. Se confirmó que los estreptococos $\beta$-hemolíticos eran $S$. pyogenes usando la prueba de pirrolidonilaminopeptidasa (PYR) (Remel, EE. UU.) según las instrucciones del fabricante. El segundo hisopo se utilizó para realizar la PRDA AlereTM TestPack Strep A, que es un ensayo inmunocromatográfico para la detección cualitativa del antígeno estreptocócico del grupo A. Una PRDA positiva o desarrollo de $S$. pyogenes en el cultivo faríngeo se consideró un diagnóstico verdadero de FE y aquellos pacientes cuyos test diagnósticos dieron resultado positivo fueron contactados por teléfono para asegurar un tratamiento antimicrobiano adecuado.

\section{Análisis de los datos}

Los datos clínicos obtenidos a partir del formulario y los resultados de las pruebas de laboratorio se vincularon con el número de identificación nacional y se ingresaron en una base de datos. Se calcularon las frecuencias de las variables de estudio. La presencia de signos y síntomas se comparó entre los niños con FE y aquellos con una PRDA o cultivo faríngeo negativo, y se calculó el chi-cuadrado para las diferentes variables de estudio con el objetivo de identificar predictores de FE. Los modelos de regresión logística se utilizaron para identificar predictores de FE, y las curvas de característica operativa del receptor (ROC) y el área bajo la curva ROC (ABC - usanza anglosajona AUC) se usaron para comparar la capacidad predictora de los modelos. Un valor P menor que 0,05 se consideró estadísticamente significativo. El análisis de los datos se realizó con el programa informático SPSS 19.2.7

\section{Resultados}

Participaron 318 niños en el estudio, de los cuales $49,7 \%$ fueron varones. La mayoría de los pacientes consultaron durante el otoño y el verano. La mediana de edad de los pacientes fue de 6,9 años (desviación estándar: $\pm 3,4$ años). Los signos y síntomas más frecuentes registrados en los formularios de estudio fueron fiebre $(86,8 \%)$, odinofagia (83\%) y exudado amigdalino $(60 \%)$ (Tabla 1). El 23,9\% (73 casos) de los pacientes fueron diagnosticados con FE.

Las características clínicas asociadas con FE en el análisis univariado fueron las siguientes: odinofagia $(\mathrm{p}=0,007)$, petequias palatinas $(\mathrm{p}<0,001)$ y estación del año (otoño/invierno; $\mathrm{p} \leq 0,001$ ). Como se indicó anteriormente en otros estudios ${ }^{20}$, encontramos que la FE fue más frecuente en otoño e invierno, incluso después de ajustar por el número de consultas en cada temporada. La fiebre se asoció inversamente con FE $(\mathrm{p}=0,002)$ (Tabla 2).

En el análisis multivariado, la odinofagia y las petequias palatinas se asociaron de forma independiente con

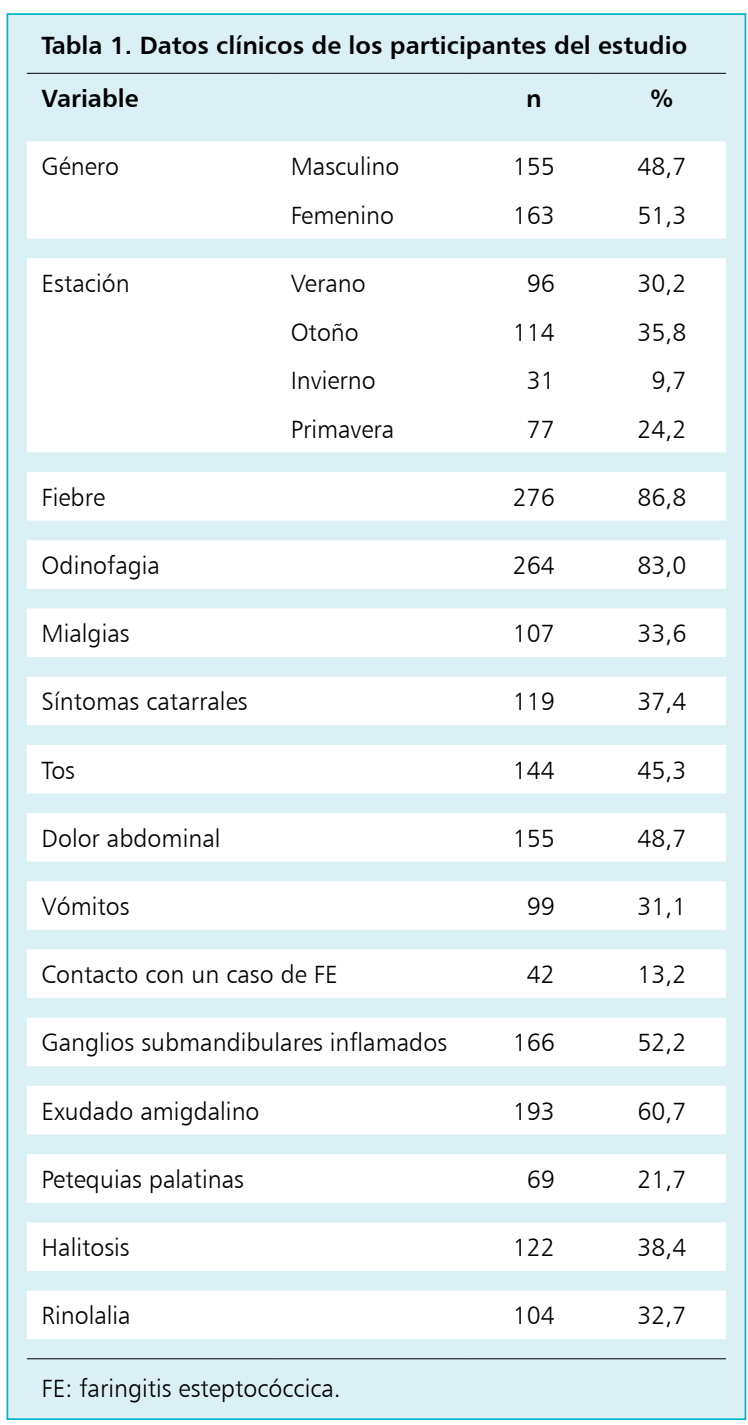

FE y se incluyeron en el modelo, pero con una capacidad predictiora baja $[\mathrm{ABC}=0,696$; IC 95\%: 0,627-0,765]. Se evaluaron diferentes modelos, ajustando el número de consultas en el Departamento de Emergencia, la edad y agrupando el otoño y el invierno en una sola variable, pero sin variación en la capacidad predictora lograda (Tabla 3 y Figura 1). Además, se ingresaron diferentes combinaciones de las variables clínicas en los análisis de regresión; sin embargo, no se incluyeron en el modelo (datos no mostrados). Aunque las asociaciones de predictores clínicos con FE fueron estadísticamente significativas, sus índices de probabilidad fueron bastante bajos, y al agruparlos en una RPC no se agregó valor diagnóstico alguno, según lo estimado por el $\mathrm{ABC}$ a partir de los modelos (Figura 1). 


\begin{tabular}{|c|c|c|c|c|c|c|c|c|c|c|}
\hline Variable & & $\begin{array}{l}\mathrm{FE} \\
(\mathrm{n})\end{array}$ & $\begin{array}{c}\text { F no } E \\
\text { (n) }\end{array}$ & Valor $p$ & $\begin{array}{c}S(\%) \\
{[95 \% \text { IC] }}\end{array}$ & $\begin{array}{c}E(\%) \\
{[95 \% \text { IC] }}\end{array}$ & $\begin{array}{l}\text { VPP (\%) } \\
\text { [95\% IC] }\end{array}$ & $\begin{array}{l}\text { VPN (\%) } \\
\text { [95\% IC] }\end{array}$ & $\begin{array}{c}\text { LR (+) } \\
{[95 \% \text { IC] }}\end{array}$ & $\begin{array}{c}\text { LR (-) } \\
{[95 \% \text { IC] }}\end{array}$ \\
\hline \multirow[t]{2}{*}{ Fiebre } & Presente & 58 & 218 & 0,002 & \multirow{2}{*}{$\begin{array}{c}76,3 \\
{[65,6-84,5]}\end{array}$} & \multirow{2}{*}{$\begin{array}{c}9,9 \\
{[6,8-14,3]}\end{array}$} & \multirow{2}{*}{$\begin{array}{c}21,0 \\
{[16,6-26,2]}\end{array}$} & \multirow{2}{*}{$\begin{array}{c}57,1 \\
{[42,2-70,9]}\end{array}$} & \multirow{2}{*}{$\begin{array}{c}0,85 \\
{[74,2-96,7]}\end{array}$} & \multirow{2}{*}{$\begin{array}{c}2,39 \\
{[1,37-4,16]}\end{array}$} \\
\hline & Ausente & 18 & 24 & & & & & & & \\
\hline \multirow[t]{2}{*}{ Odinofagia } & Presente & 71 & 193 & 0,007 & \multirow{2}{*}{$\begin{array}{c}93,4 \\
{[85,5-97,2]}\end{array}$} & \multirow{2}{*}{$\begin{array}{c}19,9 \\
{[15,4-25,4]}\end{array}$} & \multirow{2}{*}{$\begin{array}{c}26,9 \\
{[21,9-32,5]}\end{array}$} & \multirow{2}{*}{$\begin{array}{c}90,6 \\
{[79,7-95,9]}\end{array}$} & \multirow{2}{*}{$\begin{array}{c}1,17 \\
{[1,07-1,27]}\end{array}$} & \multirow{2}{*}{$\begin{array}{c}0,33 \\
{[1,21-6,73]}\end{array}$} \\
\hline & Ausente & 5 & 48 & & & & & & & \\
\hline \multirow[t]{2}{*}{ Petequias } & Presente & 28 & 41 & $<0,001$ & \multirow{2}{*}{$\begin{array}{c}36,8 \\
{[26,9-48,1]}\end{array}$} & \multirow{2}{*}{$\begin{array}{c}82,9 \\
{[77,6-87,2]}\end{array}$} & \multirow{2}{*}{$\begin{array}{c}40,58 \\
{[29,8-52,4]}\end{array}$} & \multirow{2}{*}{$\begin{array}{c}80,57 \\
{[75,2-85]}\end{array}$} & \multirow{2}{*}{$\begin{array}{c}2,16 \\
{[1,44-3,24]}\end{array}$} & \multirow{2}{*}{$\begin{array}{c}0,76 \\
{[0,64-0,91]}\end{array}$} \\
\hline & Ausente & 48 & 199 & & & & & & & \\
\hline \multirow[t]{2}{*}{ Otoño-Invierno } & Sí & 48 & 97 & $<0,001$ & \multirow{2}{*}{$\begin{array}{c}63,2 \\
{[52,3-74]}\end{array}$} & \multirow{2}{*}{$\begin{array}{c}59,9 \\
{[53,7-66,09]}\end{array}$} & \multirow{2}{*}{$\begin{array}{c}33,1 \\
{[25,4-40,78]}\end{array}$} & \multirow{2}{*}{$\begin{array}{c}83,8 \\
{[78,3-89-3]}\end{array}$} & \multirow{2}{*}{$\begin{array}{c}1,57 \\
{[1,25-1,98]}\end{array}$} & \multirow{2}{*}{$\begin{array}{c}0,61 \\
{[0,45-0,83]}\end{array}$} \\
\hline & No & 28 & 145 & & & & & & & \\
\hline
\end{tabular}

\begin{tabular}{|c|c|c|c|}
\hline Modelo & $\begin{array}{l}\text { Variables } \\
\text { incluidas }\end{array}$ & $\begin{array}{c}\text { Coeficiente } \\
\text { Beta }\end{array}$ & $\begin{array}{c}A B C \\
\text { (IC 95\%) }\end{array}$ \\
\hline \multirow[t]{5}{*}{1} & Petequias & 1,414 & \multirow{5}{*}{$\begin{array}{c}0,696 \\
(0,627-0,765)\end{array}$} \\
\hline & Odinofagia & 1,063 & \\
\hline & Otoño & 0,942 & \\
\hline & Invierno & 0,747 & \\
\hline & Primavera & 0,290 & \\
\hline \multirow[t]{2}{*}{2} & Petequias & 0,982 & \multirow{2}{*}{$\begin{array}{c}0,673 \\
(0,602-0,743)\end{array}$} \\
\hline & Odinofagia & 1,196 & \\
\hline \multirow[t]{4}{*}{3} & Petequias & 1,062 & \multirow{4}{*}{$\begin{array}{c}0,716 \\
(0,649-0,784)\end{array}$} \\
\hline & Edad & 0,122 & \\
\hline & Mialgias & $-0,666$ & \\
\hline & Odinofagia & 0,978 & \\
\hline \multirow[t]{4}{*}{4} & Petequias & 1,049 & \multirow{4}{*}{$\begin{array}{c}0,707 \\
(0,636-0,778)\end{array}$} \\
\hline & Otoño-Invierno & 0,781 & \\
\hline & Edad & 0,127 & \\
\hline & Mialgias & $-0,717$ & \\
\hline \multicolumn{4}{|c|}{$\begin{array}{l}\text { FE: faringitis estreptocóccica. Modelo 1: No ajustado. Mode- } \\
\text { lo 2: Ajustado por el número de consultas. Modelo 3: La edad } \\
\text { fue agregada como como una posible variable explicativa. } \\
\text { Modelo 4: La estación del año fue cambiada a una variable } \\
\text { dicotómica agrupando otoño e invierno y fue agregada como } \\
\text { como una posible variable explicativa. }\end{array}$} \\
\hline
\end{tabular}

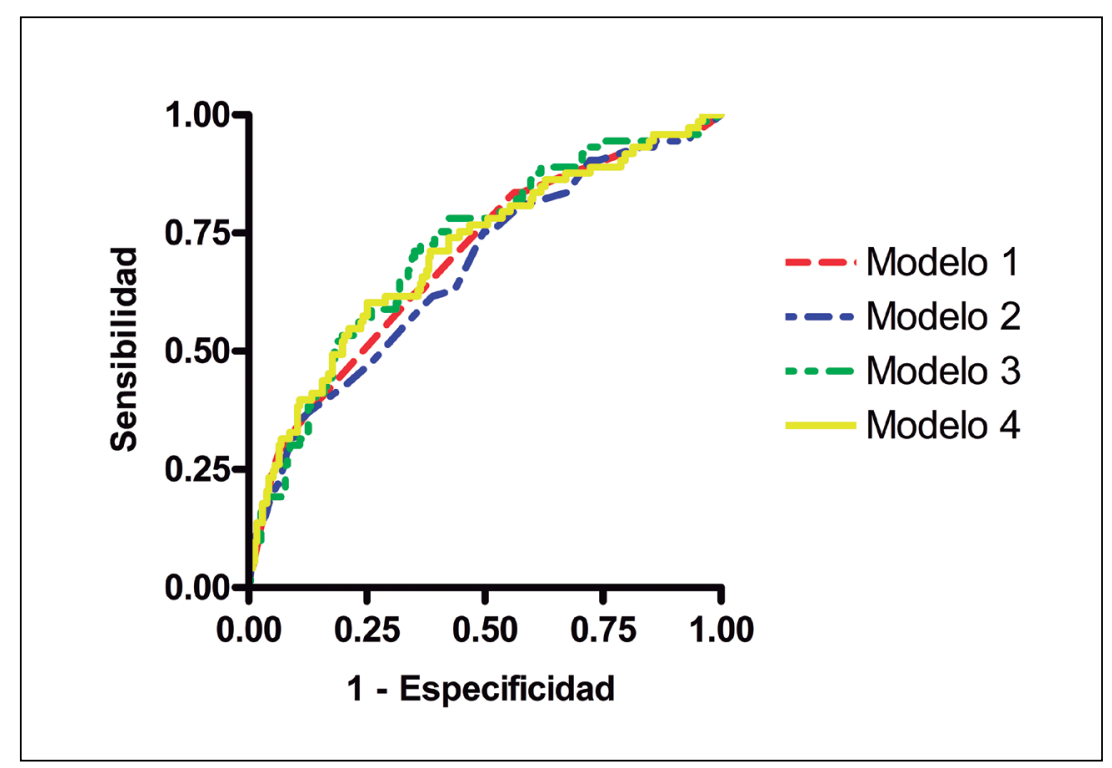

Figura 1. Curvas ROC de los modelos 1-4 descritos en la Tabla 3.

\section{Discusión}

Nuestros resultados arrojaron asociaciones estadísticamente significativas de variables clínicas con FE (odinofagia, petequias palatinas, estación del año y fiebre) en el análisis univariado. Es de destacar que la fiebre se asoció inversamente con la etiología estreptocóccica, aunque se ha informado previamente que la fiebre se asoció positivamente con FE e incluso se 
ha incluido en otras $\mathrm{RPC}^{23}$. La causa puede ser que la fiebre es un síntoma muy inespecífico y frecuente en la faringitis viral.

En el análisis multivariado, la fiebre no mostró asociación con FE; sin embargo, las petequias palatinas, la odinofagia y la estación fría aún se incluyeron en la mayoría de los modelos como predictores independientes de FE. Hasta donde sabemos, estudios recientes no han incluido las petequias palatinas, que fue el signo más consistente incluido en todos los modelos. Al considerar la edad, el dolor muscular parece ser una variable asociada de forma independiente con la faringitis no estreptocóccica, probablemente porque los niños más pequeños pueden no describir este síntoma muy claramente en comparación con los niños mayores.

La exactitud del juicio clínico para el diagnóstico de FE ha sido ampliamente discutida en la literatura médica. La mayoría de los autores han concluido que los hallazgos clínicos no son confiables para un diagnóstico preciso de FE. Shaikh y cols. ${ }^{8}$, realizaron una revisión sistemática de la precisión de los signos y síntomas de FE en niños, y también incluyeron estudios con RPC. Estos autores concluyeron que las características clínicas, ya fuera individualmente o combinadas en las reglas de predicción, no se pueden utilizar para diagnosticar o excluir definitivamente la FE. La última afirmación podría ser cierta en entornos con pruebas de confirmación microbiológica disponibles, junto con una baja incidencia de complicaciones de la FE. Sin embargo, la situación podría ser diferente en entornos de bajos recursos donde el umbral de tratamiento es más bajo, lo que da a las RPC para FE un papel potencialmente más importante. En Chile, las pruebas de diagnóstico microbiológico no están disponibles en muchos centros de atención de la salud, especialmente en los entornos públicos de atención primaria de salud. Por lo tanto, la pregunta de si una RPC para FE podría ayudar a reducir el tratamiento antimicrobiano innecesario es absolutamente válida.

Si bien Chile es un país en desarrollo, se observa una baja incidencia de complicaciones no supurativas. De hecho, de acuerdo con el sistema nacional de registro de altas hospitalarias, no se informó caso alguno de complicaciones no supurativas en niños bajo 9 años de edad, y sólo se informaron siete casos en el grupo de 10 a 19 años en $2009^{22}$

La otra preocupación principal del uso excesivo de antimicrobianos es la resistencia a ellos y también se ha documentado en nuestro país (a mcrólidos). De acuerdo con la vigilancia epidemiológica nacional de las infecciones por $S$. pyogenes publicado por el Instituto de Salud Pública de Chile, la susceptibilidad a la penicilina fue de $100 \%{ }^{23}$. Esto está en gran contraste con países como India, donde $23,6 \%$ de los aislados de $S$. pyogenes tienen una susceptibilidad intermedia a penicilina ${ }^{24}$. Finalmente, no existen datos con respecto a las reacciones adversas derivadas del uso innecesario de antimicrobianos.

Fischer y cols. (2006), compararon la efectividad de siete RPC para el manejo de niños egipcios con FE, y evaluar así la utilidad de las RPC en entornos carentes de pruebas de laboratorio. Sus resultados revelaron que el uso de reglas de mejor rendimiento disminuiría el uso innecesario de antimicrobianos en 31 a 40\%, en comparación con la estrategia de tratar presuntivamente todos los casos de faringitis clínica. No obstante, la estrategia de la regla trataría hasta $60 \%$ de los casos de cultivos negativos. También encontraron diferencias sustanciales entre los valores informados de sensibilidad, especificidad, valores predictores y razones de verosimilitud al aplicar las reglas en una población diferente de aquella en la que se derivó la regla, destacando la importancia de la validación local apropiada.

Se ha indicado anteriormente que las RPC para pacientes pediátricos tienen una calidad inferior a las de los adultos debido a obstáculos particulares en la investigación pediátrica, como una mayor variabilidad inter-observador en la historia y el examen físico, un bajo número de niños con el trastorno que se quiere estudiar y resultados poco $\operatorname{precisos}^{25}$. Las deficiencias de calidad metodológica informadas con mayor frecuencia son un cegamiento inadecuado, una evaluación limitada de la reproducibilidad de las variables predictoras, un seguimiento inadecuado para evaluar los resultados de los pacientes y un poder estadístico insuficiente. El presente estudio utilizó los signos y síntomas más ampliamente descritos ${ }^{8}$ y aseguró todas las medidas de resguardos metodológicos para reducir estas deficiencias. Sin embargo, no fue capaz de desarrollar una regla clínica que pudiera incluir o excluir con seguridad la etiología estreptocóccica.

Una posible limitación del presente estudio es la falta de evaluación de la reproducibilidad de las variables predictoras. Sin embargo, esto también puede representar mejor el "mundo real" de la atención médica donde existe una amplia variabilidad inter-observador al diagnosticar FE.

El presente estudio realizó un intento de alta calidad metodológica para obtener una RPC para el diagnóstico de FE. Los resultados obtenidos nos permiten afirmar con certeza que los hallazgos clínicos no pueden utilizarse para diagnosticar FE en niños, incluso aquellos incluidos sistemáticamente en RPC para el tratamiento de FE en niños. Sin embargo, podría haber una utilidad para las RPC para FE en entornos específicos, de acuerdo con los diferentes umbrales de tratamiento, la disponibilidad de pruebas de diagnóstico y la carga de complicaciones derivadas de los casos de FE no tratados. El papel potencial de las RPC para FE debe estudiarse en cada entorno. Particularmente en nuestro entorno, el poder predictor de los modelos evaluados es inaceptablemente bajo, por lo 
que es necesario y urgente mejorar el acceso a las pruebas de confirmación en nuestro país.

Agradecimientos. Al personal del Laboratorio de Microbiología de la Pontificia Universidad Católica de Chile por su ayuda en los aspectos técnicos de este trabajo. Queremos agradecer a Lorena Silva por la coordinación logística de este trabajo.

\section{Resumen}

Introducción: La etiología estreptocóccica de una faringitis debe ser confirmada con métodos de laboratorio para evitar un sobre-tratamiento antimicrobiano, exámenes que agregan costo a la atención del paciente. Los scores diseñados para aplicar en niños y adultos son imperfectos. Objetivo: Desarrollar una regla de predicción clínica para contribuir al diagnóstico de la faringitis estreptocóccica (FE) en niños. Pacientes y Métodos: Se incluyeron 318 pacientes de 2 a 15 años que fueron eva- luados por sospecha de FE en el Servicio de Urgencias Pediátricas y la Unidad de Pediatría Ambulatoria de la Red Salud UC-Christus. Se obtuvo un cultivo faríngeo y una prueba rápida de detección de antígeno para Streptococcus pyogenes de cada paciente. Los datos se analizaron para posibles predictores clínicos de FE con análisis de regresión múltiple. Resultados. Setenta y tres casos de FE fueron diagnosticados (23,9\%). En el análisis univariado, la fiebre se asoció inversamente con FE $(p=0,002)$. La odinofagia, las petequias palatinas y la estación del año (otoño e invierno) se asociaron positivamente con $\mathrm{FE}$ ( $p=0,007, p<0,001$ y $p=0,03$ respectivamente). En el análisis de regresión múltiple, los modelos no tuvieron suficiente poder para predecir etiología por $S$. pyogenes. Conclusión: Los predictores clínicos analizados, incluso los incluidos sistemáticamente en reglas de predicción clínica, no mostraron suficiente poder predictor para incluir o excluir de forma segura la $\mathrm{FE}$ en este contexto y, por lo tanto, sería necesario mejorar el acceso a las pruebas de confirmación.

\section{Referencias bibliográficas}

1.- Capurro D, Rada G. The diagnostic process. Rev Med Chile 2007; 135: 534-8. http://dx.doi. org/10.4067/S0034-98872007000400018.

2.- Ferrero F, Nascimento-Carvalho C M. Clinical prediction rules and pediatric infectious ispidiseases. Pediatr Infect Dis J 2012; 31: 628-9. doi: 10.1097/ INF.0b013e31825670f2.

3.- Bisno A L. Acute pharyngitis: etiology and diagnosis. Pediatrics 1996; 97: 949-54.

4.- Castillo M, Morales V, Fonseca X, Cifuentes L, García P, Catalán S, et al. Absence of correlation between clinical variables and etiology of acute pharyngotonsillitis: Prospective study of cases and controls. Rev Otorrinolaringol Cir Cabeza Cuello 2008; 68: 7-15.

5.- Muñoz S, Córdova M, Morales V, Cifuentes L. Sore throat, ¿streptococcal etiology? Use of etiological screening and antimicrobial prescription in two university pediatric centers. Rev Chil Infect 2005; 22: 147-53. DOI: /S071610182005000200005.

6.- Sims Sanyahumbi A, Colquhoun S, Wyber R, Carapetis J. Global disease burden of Group A Streptococcus. In: Streptococcus pyogenes: Basic Biology to Clinical Manifestations. Editores: Joseph J. Ferretti, Dennis L. Stevens, Vincent A. Fischetti. Oklahoma, OK: The University of Oklahoma Health Sciences Center 2016; 480-500.

7.- Del Mar C B, Glasziou P P, Spinks A B. Antibiotics for sore throat. Cochrane Database Syst Rev 2006; CD000023.
DOI: 10.1002/14651858.CD000023.pub3.

8.- Shaikh N, Swaminathan N, Hooper E G. Accuracy and precision of the signs and symptoms of streptococcal pharyngitis in children: a systematic review. J Pediatr 2012; 160: 487-93. doi: 10.1016/j. jpeds.2011.09.011.

9.- Gerber M A, Baltimore R S, Eaton C B, Gewitz M, Rowley A H, Shulman S T, et al. Prevention of rheumatic fever and diagnosis and treatment of acute streptococcal pharyngitis: a scientific statement from the American Heart

Association Rheumatic Fever, Endocarditis and Kawasaki Disease Committee of the Council on Cardiovascular Disease in the Young, the Interdisciplinary Council on Functional Genomics and Translational Biology, and the Interdisciplinary Council on Quality of Care and Outcomes Research: endorsed by the American Academy of Pediatrics. Circulation 2009; 119: 1541-51. DOI:10.1161/ CIRCULATIONAHA.109.191959.

10.- Bisno A L, Gerber M A, Gwaltney J M Jr, Kaplan E L, Schwartz R H. Practice guidelines for the diagnosis and management of group A streptococcal pharyngitis. Clin Infect Dis 2002; 35: 113-25. DOI: 10.1086/340949.

11.- Chiappini E, Regoli M, Bonsignori F, Sollai S, Parretti A, Galli L, et al. Analysis of different recommendations from international guidelines for the management of acute pharyngitis in adults and children. Clin Ther 2011; 33: 48-58. doi: 10.1016/j.clinthera.2011.02.001.

12.- Steinhoff M C, Fischer Walker C, Rimoin A W, Hamza H S. A clinical decision rule for management of streptococcal pharyngitis in low-resource settings. Acta Peadiatr 2005; 94 : 1038-42. DOI:10.1080/08035250510027994.

13.- McIsaac W J, White D, Tannenbaum D, Low D E. A clinical score to reduce unnecesary antibiotic use in patients with sore throat. Can Med Assoc J 1998; 158: 75-83.

14.- Attia M W, Zaoutis T, Klein J D, Meier F A. Performance of a predictive model for streptococcal pharyngitis in children [la corrección aparece publicada en Arch Pediatr Adolesc Med 2001; 155 (10): 1179] Arch Pediatr Adolesc Med 2001; 155 (6): 687-91.

15.- Smeesters P R, Campos D Jr, Van Melderen L, de Aguiar E, Vanderpas J, Vergison A. Pharyngitis in low-resources settings: a pragmatic clinical approach to reduce unnecessary antibiotic use. Pediatrics 2006; 118: e1607-11. DOI: 10.1542/peds.20061025.

16.- Joachim L, Campos D Jr, Smeesters P R. Pragmatic scoring system for pharyngitis in low-resources settings. Pediatrics 2010; 126(3): e608-14. doi: 10.1542/peds.2010-0569.

17.- Fischer C, Rimoin A, Hamza H, Steinhoff M. Comparison of clinical prediction rules for management of pharyngitis in settings with limited resources. J Pediatr 2006: 149: 64-71. DOI: 10.1016/j.jpeds.2006.03.005.

18.- Freeman D H. Applied categorical data analysis. Marcel Dekker Inc., New York 1987. ISBN:0-824-77752-2.

19.- Peduzzi P, Concato J, Kemper E, Holford T R, Feinstein A R. A simulation study of the number of events per variable in logistic regression analysis. J Clin Epidemiol 1996: 49 (12): 1373-9. 
20.- McGinn T, Wyer P, Wisvinesky J, Deveraux P, Stiell I, Richardson S, et al. Clinical Prediction Rules. En: Users' Guides to thesepicical Literature. Editores: Guyatt G, Rennie D, Meade M, Cook D, Mc Graw Hill 2008. 491505.

21.- Gerber M A. Diagnosis and treatment of pharyngitis in children. Pediatr Clin North Am 2005; 52: 719-47.

22.- Stjernquist-Desatnik A, Orrling A.
Pharyngotonsillitis. Periodonthology 2009; 49: 140-50.

23.- Instituto de Salud Pública de Chile (2013) Boletín ISP: Vigilancia de Laboratorio de la enfermedad invasora causada por Streptococcus pyogenes, Chile 2009-2013. 25 de Abril 2017: http://www.ispch.cl/sites/default/files/ Boletin\%20S\%20pyogenes\%2030-12-2013_0. pdf.

24.- Ray D, Saha S, Sinha S, Kumar Pal N,
Bhattacharya B. Molecular characterization and evaluation of the emerging antibiotic-resistant Streptococcus pyogenes from Eastern India. BMC Infectious Diseases 2016; 16: $753 \mathrm{https://}$ doi.org/10.1186/s12879-016-2079-9.

25.- Maguire J L, Kulik D M, Laupacis A, Kuppermann N, Uleryk E, Parkin P. Clinical prediction rules for children: A systematic review. Pediatrics 2011; 128: e666-e677. doi: 10.1542/peds.2011-0043. 